\title{
Le différentiel de sélection multiple
}

\author{
Louis Laurencelle $\mathrm{a}^{\mathrm{a}, \mathbb{\triangle}}$ \\ ${ }^{\text {a}}$ Université du Québec à Trois-Rivières
}

\begin{abstract}
An important aim of personnel selection is to maintain and hopefully increase the company's productivity, a similar rationale underlying the recruiting of athletes in a sports league or the admission of students or candidates in limited enrolment programs. To help raise the 'performance' of the organization, one of many ways is to submit candidates to an aptitude test and select only those whose score exceeds some predetermined threshold value. The eventual 'performance' of such abler candidates should be superior to that of un-selected personnel, inasmuch as there is a positive relation between performance in the organization and the score obtained at the aptitude test. The expected gain in performance, i.e. the positive score difference between the sub-population of selected and abler persons and the whole pool of candidates is called 'selection differential', a very old concept. Brogden, in 1951, introduced the idea of differential selection in the specific sense of testing candidates on two or more aptitude tests in order to assign each meritorious candidate to the job wherein the expected gain is higher. Taking up this idea, we propose and develop the concept and mathematics of the multivariate selection differential, with three variants. A few worked-out examples and tables of differentials of order 2 to 5 complete the paper.
\end{abstract}

Keywords - Personnel selection; selection differential; multivariate selection differential; differential selection; gain in performance.

louis.laurencelle@gmail.com

\section{Introduction}

Dans les grandes entreprises comme l'Armée américaine, la fonction publique, les compagnies et industries nationales et multinationales, la sélection du personnel est une affaire sérieuse. Qu'il s'agisse de nouvelles recrues potentielles ou d'employés demandant une réaffectation, les candidats à l'emploi sont soumis à une procédure plus ou moins définie : entrevues, tests d'aptitudes, examens physiques et psychologiques divers, puis une décision est prise à leur endroit : "retenu dans l'emploi" ou "refusé". L'application sérieuse d'une procédure de sélection dépend bien sûr de l'impact que la sélection peut avoir sur l'entreprise. L'une des dimensions de cet impact est la productivité de la personne retenue dans son nouvel emploi.

Le différentiel de sélection peut être défini comme le gain de productivité escompté grâce à la sélection du ou des meilleurs candidats dans un emploi. Sélection pour un emploi, admission dans un programme d'études, affectation ou orientation dans différentes fonctions sont autant de situations auxquelles on peut appliquer le concept de différentiel de sélection. S'il semble préférable d'employer quelqu'un de mieux qualifié, c'est qu'on espère que la performance à l'emploi de cette personne sera supérieure à celle d'une personne prise au hasard, sans sélection. Cette différence de performance, soit le différentiel de sélection
Dy, dépend de différents paramètres et peut être exploitée dans un contexte d'emplois multiples auxquels sont assorties autant de règles de sélection correspondantes. C'est ainsi qu'apparaît le concept de différentiel de sélection multiple, notamment dans ses deux formes standards, soit le différentiel multiple, $\Delta_{k, \rho}(P)$, et le différentiel polyvalent, $\Delta_{k, \rho}^{p}(P)$.

Après avoir recensé les rares articles publiés sur la question, incluant les écrits mathématiques utiles pour l'évaluation numérique du différentiel de sélection, nous identifierons en détail le concept général et ses formes standards. Nous développerons ensuite le concept du différentiel de sélection multiple en présentant un théorème permettant de le quantifier. Nous passerons en revue les méthodes d'intégration, d'évaluation numérique et d'estimation mises en oeuvre pour le calcul des différentiels : ce calcul sera illustré en détail pour un cas particulier. Nous présenterons enfin des tableaux de différentiels standards correspondant à différentes combinaisons de conditions et nous conclurons sur la question.

\section{Documentations}

Les concepts de différentiel et de différentiel de sélection sont suffisamment anciens pour décourager toute velléité de repérage historique. Le différentiel de sélection (simple) désigne tout simplement la différence entre la "va- 
leur" moyenne des membres d'un groupe sélectionné et celle de la population générale, soit

$$
D_{y}=\overline{\mathrm{X}}_{s}-\mu_{x}
$$

cette différence, qu'on suppose positive, constitue l'avantage, voire la raison d'être de la sélection.

L'idée et la mathématique élémentaire du différentiel de sélection sont apparues au moins dès 1946, dans un article de BRogden (1946). Ce dernier, parlant de sélection pour un emploi à partir d'un test, montre que l'avantage de sélection dépend directement du coefficient de validité prédictive (disons $\rho_{x, y}$ ) plutôt que de son carré $\left(\rho^{2}\right)$, par exemple, et que cet avantage peut être mesuré.

L'avantage de sélection, en forme standardisée et en espérance, peut s'écrire :

$$
\left.\Delta=E\left\{\overline{\mathrm{X}}_{S}-\mu_{x}\right) / \sigma_{\mathrm{X}}\right\}
$$

cette quantité est connue sous différents noms, notamment celui de différentiel de sélection (standard). Pour tout modèle de distribution, sa valeur peut être déterminée, le modèle habituellement invoqué étant celui de la distribution normale des X. BURRows $(1972,1975)$ a étudié l'espérance et la variance de $\Delta$ dans des cas d'échantillons finis; NAGARAJA (1982) a considéré d'autres aspects limites de $\Delta$.

BROGDEN (1951) semble être le premier et le seul à avoir développé l'idée d'un avantage spécifique de la sélection différentielle. ${ }^{1}$ Dans son long article, il considère une situation dans laquelle des candidats pour $k$ emplois sont évalués au moyen de $k$ systèmes sélectifs, chaque système ayant un coefficient de validité prédictive donné $\rho\left(x_{j}, y_{j}\right)$ pour l'emploi correspondant, et les systèmes prédictifs ayant entre eux des relations d'inter-corrélation connues. Il développe en détail, quoique gauchement étant donné l'époque, la solution mathématique du cas de deux emplois $(k=2)$. C'est ce qui donne lieu au concept que nous proposons ici, le différentiel de sélection multiple, que nous reprenons de Brogden en le généralisant pour tout $k \geq 2$ et que nous tentons d'asseoir sur une base mathématique plus moderne.

Le concept de différentiel de sélection, qu'il soit simple ou multiple, n'est qu'un des aspects d'une stratégie, parmi de multiples stratégies disponibles aux personnes intéressées au recrutement, à la sélection et à l'orientation des ressources. D'autres auteurs ont traité de ces sujets, notamment pour la sélection d'élèves dans des programmes d'études (FInNEY, 1962; DunNETT, 1978). Nous nous restreindrons ici au seul aspect, très partiel, du différentiel de sélection.

L'évaluation numérique des différentiels de sélection, sous l'hypothèse d'une distribution normale et multinor- male des critères de performance à l'emploi, pose certains problèmes pratiques de calcul. Nous avons puisé ce que nous avons pu principalement dans le traité de JOHNSON, KotZ et BALAKRISHNAN (2000), mais aussi dans DAVID (1981) et d'autres publications ad hoc citées par ceuxlà. Nous avons dû élaborer nous-même une part des solutions requises, certaines exactes et d'autres approximatives : nous en ferons état le moment venu.

\section{Analyse du concept}

Supposons qu'il existe une population de personnes admissibles dans un emploi et que la performance de chacun dans l'emploi, sa productivité, est indiquée numériquement par la variable Y. Si l'entreprise ne sélectionne pas ceux qu'elle emploie mais les engage au hasard, la performance dans l'entreprise variera autour d'une moyenne $\mu_{\mathrm{Y}}$ avec un écart-type $\sigma_{Y}$, paramètres qui reflètent la composition de la population des personnes admissibles.

Supposons dorénavant que, dans l'espoir d'augmenter sa productivité, l'entreprise procède à la sélection de ses recrues au moyen d'un test ou d'un système de sélection particulier. La mesure de chaque candidat au test est dénotée $\mathrm{X}$, avec $\mu_{\mathrm{X}}$ et $\sigma_{\mathrm{X}}$ comme paramètres dans la même population de base. Pour qu'il serve à quelque chose, le test doit entretenir avec la performance à l'emploi une certaine relation positive, reflétée par une corrélation linéaire $\rho_{X, Y}$ telle que $\rho_{\mathrm{X}, \mathrm{Y}}>0$. La sélection consiste donc à retenir pour l'emploi les meilleurs candidats au test. Quel sera l'impact de cette sélection sur l'entreprise?

La détermination du nombre $N$ d'individus retenus pour l'emploi peut se faire selon deux règles distinctes : retenir ceux qui occupent la proportion $P$ supérieure de la population, ou bien retenir les $\mathrm{N}$ meilleurs candidats parmi ceux qui se présentent au test, quelle que soit la place qu'occupent leurs scores dans la population. Bien qu'elle soit plausible et sans doute pratiquée, nous ne considérerons pas cette seconde option, dont le rendement pour l'entreprise reste presque impossible à anticiper puisqu'il dépend étroitement de la cohorte des candidats qui se présentent au test.

Donc, dans le cas d'un test, quantifié par X, visant à prédire la performance $Y$ dans un emploi, on peut admettre pour chaque individu la relation linéaire suivante entre performance prédite à l'emploi ( $\left.\mathrm{Y}^{\prime}\right)$ et résultat au test $(\mathrm{X})$ :

$$
\begin{aligned}
Y^{\prime} & =f(X) \\
& =\frac{\sigma_{\mathrm{Y}}}{\sigma_{\mathrm{X}}} \rho_{\mathrm{X}, \mathrm{Y}} \mathrm{X}+\mu_{\mathrm{Y}}-\frac{\sigma_{\mathrm{Y}}}{\sigma_{\mathrm{X}}} \rho_{\mathrm{X}, \mathrm{Y}} \mu_{\mathrm{X}} .
\end{aligned}
$$

Le gain de performance espéré se mesure par la différence entre $\overline{\mathrm{Y}}_{s}$, la moyenne de performance des $N$ individus sé-

1. D'autres auteurs néanmoins ont cité Brogden, notamment JARret (1948), Brown et GHiSELLi (1953), voire ANASTASI (1988), les citations étant cependant floues et incomplètes. 
lectionnés, et $\mu_{\mathrm{Y}}$, la performance moyenne, ou typique, de la population concernée. La quantité $\overline{\mathrm{X}}$ désignant le score moyen au test pour les individus sélectionnés, le différentiel de sélection est alors :

$$
\begin{aligned}
D_{y} & =\overline{\mathrm{Y}}_{s}-\mu_{y} \\
& =\rho_{\mathrm{X}, \mathrm{Y}} \sigma_{\mathrm{Y}} \frac{\overline{\mathrm{X}}_{\mathrm{s}}-\mu_{\mathrm{X}}}{\sigma_{\mathrm{X}}}
\end{aligned}
$$

L'analyse de cette fonction montre que l'avantage de sélection est proportionnel (1) au coefficient de validité prédictive $\rho_{\mathrm{X}, \mathrm{Y}}$, (2) à l'écart-type $\sigma_{\mathrm{Y}}$, ou variabilité, des mesures de performance, et (3) à la différence standardisée des scores obtenus par les individus retenus au test. À coefficient de validité et écart-type de performance constants, l'avantage de sélection va dépendre essentiellement du troisième facteur, la différence $\left(\overline{\mathrm{X}}_{s}-\mu_{\mathrm{X}}\right) / \sigma_{\mathrm{X}}$. L'espérance de cette quantité, c'est-à-dire sa valeur attendue d'un échantillon à l'autre, est le différentiel de sélection simple, en forme standard, soit :

$$
\Delta(P)=E\left\{\left(\overline{\mathrm{X}}_{s}-\mu_{\mathrm{X}}\right) / \sigma_{\mathrm{X}}\right\},
$$

où $P$ indique le taux de sélection, avec $0 \leq P \leq 1$. S'il n'y a pas de sélection, c'est-à-dire si $P \rightarrow 1$, alors chaque individu est pris comme au hasard et les scores $\mathrm{X}$ des candidats gravitent autour de la moyenne $\mu_{\mathrm{X}}$, avec $\Delta(1)=0$. Le différentiel $\Delta(P)$ dépendra donc du taux de sélection $P$, lequel correspond au complément du rang centile $(=1-P)$ au-delà duquel est opérée la sélection. En fait, la valeur du différentiel $\Delta(P)$ est une fonction inversement reliée au taux de sélection $P$. Le lecteur est référé aux ouvrages précédents (LAURENCELLE, 1994, 1998, 2008) pour un traitement spécifique et plus détaillé du différentiel de sélection simple.

Reprenons maintenant le problème mais, cette fois, recrutons des individus pour deux emplois distincts, en exploitant un test de sélection spécifique pour chaque emploi. Dans l'ensemble des candidats examinés, certains pourront se qualifier pour l'emploi 1, d'autres pour l'emploi 2, et certains peut-être pour les deux emplois. L'avantage de sélection peut ici être envisagé de deux manières, selon la procédure de recrutement appliquée. $\mathrm{Si}$, pour chaque individu qualifié dans les deux emplois, l'attribution à l'un ou l'autre emploi se fait au hasard, le différentiel de sélection pour l'entreprise sera le même que celui déjà établi dans le cas d'un seul emploi. Si, par contre, l'individu est orienté vers l'emploi pour lequel il s'est le mieux qualifié, l'avantage de sélection sera généralement accru. La valeur accrue ainsi engendrée se reflète dans le différentiel de sélection multiple, symbolisé par $\Delta_{k, \rho}(P)$ : la valeur standardisée dépend à la fois du taux de sélection $P$, mais aussi du nombre $k$ de couples test :emploi considérés, ici $k=2$ dans notre exemple, et enfin de la corrélation $(\rho)$ entre les tests utilisés. Une autre manière encore de procéder dans une entreprise consisterait à ne retenir que ceux se qualifiant dans tous les emplois, quitte à assigner chaque candidat qualifié dans l'emploi où il devrait performer le mieux : cette autre méthode se concrétise dans les différentiels de sélection polyvalents, symbolisés $\Delta_{k, \rho}^{p}(P)$.

L'incorporation de ces concepts et procédures dans une stratégie opérationnelle de recrutement et de gestion de personnel, ou de sélection académique, déborde les intentions du présent article et elle représente un certain défi. Nous simplifions donc le problème de manière drastique, en imposant par exemple, pour les cas de tests multiples, des coefficients de validité prédictive tous égaux, ce qui nous permet de les ignorer tout à fait en occultant le facteur correspondant dans l'équation (4). Nous simplifions encore le problème en supposant, pour les cas de $k=3$ tests ou plus, des inter-corrélations entre les tests toutes égales à $\rho(\rho \geq 0)$. Enfin le modèle de la distribution normale, qui demeure une hypothèse raisonnable pour la distribution des scores (standardisés) de chaque test $\mathrm{X}_{j}$, est imposé ici en tant que fiction commode.

\section{Le différentiel de sélection multiple (et polyvalent) stan- dard}

Modèle général et simplifications. Nous supposons une population de candidats de taille virtuellement infinie. Les membres de cette population sont mesurables selon chacun de $k$ tests, ou systèmes prédictifs distincts, à mesures respectives $\mathrm{X}_{1}, \mathrm{X}_{2}, \ldots, \mathrm{X}_{k}$. Sans sacrifier la généralité, nous posons que ces mesures sont données en valeurs standardisées, toutes d'espérance 0 et de variance 1 . En outre, les relations statistiques entre les tests sont toutes linéaires et de corrélations égales à $\rho$, où $\rho \geq 0$. De plus, pour chaque type d'emploi, il existe une mesure de performance $\mathrm{Y}_{j}$, $1 \leq j \leq k$, donnée aussi en valeurs standardisées, et la relation linéaire entre emploi et test correspondant est indiquée par un coefficient de validité prédictive $\rho_{X, Y}$ (où $\rho_{X, Y} \geq 0$ ), présumé égal pour tous les couples test :emploi. Notons que ces dernières simplifications, concernant les variances et les corrélations pour les emplois, sont importantes : elles affectent la généralité des résultats et sont susceptibles d'altérer la précision des calculs. Enfin, toutes les distributions statistiques sont réputées normales.

Taux de sélection souhaité versus réel. Si nous fixons à $P$ la fraction de population dans laquelle nous voulons recruter des candidats par un test dans un emploi, il convient de retenir pour l'emploi ceux dont le score $\mathrm{X}$ au test atteint et dépasse le centile 100(1-P), disons $c=\mathrm{X}_{(1-P)}$. Supposons maintenant que, pour deux emplois, nous mettions à contribution deux tests, dénotés $X_{1}$ et $X_{2}$. La sélection selon le test $\mathrm{X}_{1}$ (c'est-à-dire, pour ceux obtenant $\mathrm{X}_{1} \geq c$ ) captera une portion $\mathrm{T}$ supérieure de la population; 
de même pour le test $X_{2}$, encore avec une portion T. Toutefois, parmi ceux s'étant qualifiés pour le test $X_{1}$, quelquesuns se qualifieront aussi pour $\mathrm{X}_{2}$, la portion conjointe étant notée $\mathrm{T}_{2}$. Les individus doublement qualifiés seront identifiés puis répartis, d'une manière ou l'autre, dans chacun des emplois, chaque emploi recevant ainsi une portion effective moyenne $\mathrm{T}-\mathrm{T}_{2}+(1 / 2) \mathrm{T}_{2}=\mathrm{T}-(1 / 2) \mathrm{T}_{2}$ des individus. Comme nous voulons sélectionner une portion effective $P$ pour l'ensemble des tests, nous devons appliquer l'équation:

$$
P=2 \mathrm{~T}-\mathrm{T}_{2} \quad(k=2),
$$

qu'il faut résoudre pour $\mathrm{T}$. Or, $\mathrm{T}_{2}$, la portion de qualification commune aux deux tests, dépend de $\mathrm{T}$ (le taux de qualification dans chaque test) et de $\rho$, la corrélation entre les tests. Ainsi, si par exemple $\rho=1$, chaque individu se qualifiant dans un test le fera aussi dans l'autre, d'où $\mathrm{T}_{2}=\mathrm{T}$ et $\mathrm{T}=P$. Si, d'autre part, la corrélation entre tests est de zéro, alors $\mathrm{T}_{2} \approx \mathrm{T}^{2}$, par le théorème de la probabilité de l'occurrence conjointe de deux événements indépendants, et conséquemment $\mathrm{T}=1-\sqrt{1-P} .{ }^{2}$ Pour des valeurs intermédiaires de $\rho$, la quantité $\mathrm{T}_{2}$ peut être évaluée comme l'aire d'une distribution bivariée bornée dans le coin supérieur $\mathrm{X}_{1}>c$ et $\mathrm{X}_{2}>c$.

Pour $k \geq 2$ tests, en général, certains des individus qualifiés dans un test (prenons $\mathrm{X}_{1}$ ) peuvent se qualifier aussi dans l'un ou plusieurs des autres tests (prenons $\mathrm{X}_{2}, \mathrm{X}_{3}, \ldots, \mathrm{X}_{k}$ ), ce dans des portions communes décroissantes, de $\mathrm{T}_{2}$ (pour la qualification par 2 tests) jusqu'à $\mathrm{T}_{k}$ (pour la qualification par tous les tests) : or il faut répartir ces personnes à qualifications multiples.

Ainsi, pour $k=3$ tests, les individus 3-qualifiés (pour ainsi dire) qui représentent une fraction $\mathrm{T}_{3}$ dans la population peuvent être répartis également entre les 3 tests, et emplois, dans une fraction respective $\mathrm{T}_{3} / 3$ par emploi. Les $T_{3}$ individus 3-qualifiés ayant été répartis, il en reste $T_{2}-T_{3}$ co-qualifiés seulement par les tests 1 et 2 , et encore $T_{2}-T_{3}$ pour les seuls tests 1 et 3 , individus qu'il faut répartir aussi entre les deux emplois : dans cette catégorie, nous retenons donc $2 \times\left(\mathrm{T}_{2}-\mathrm{T}_{3}\right) / 2=\mathrm{T}_{2}-\mathrm{T}_{3}$ individus co-qualifiés par le test et assignés à l'emploi 1 . Finalement, à partir des $\mathrm{T}\left(=\mathrm{T}_{1}\right)$ individus qualifiés notamment au test 1 , la fraction résiduelle d'individus qualifiés seulement par ce test est $\mathrm{T}-2\left(\mathrm{~T}_{2}-\mathrm{T}_{3}\right)-\mathrm{T}_{3}=\mathrm{T}-2 \mathrm{~T}_{2}+\mathrm{T}_{3}$. Globalisant sur les trois tests, on arrive ainsi à :

$$
\begin{aligned}
P & =3 \times\left[-2 \mathrm{~T}_{2}+\mathrm{T}_{3}+\mathrm{T}_{2}-\mathrm{T}_{3}+\mathrm{T}_{3} / 3\right] \\
& =3 \mathrm{~T}-3 \mathrm{~T}_{2}+\mathrm{T}_{3} \quad(k=3),
\end{aligned}
$$

La fraction $\mathrm{T}\left(=\mathrm{T}_{1}\right)$ dénote la portion d'une distribution normale univariée située au delà d'un point $c, c$ étant donc le centile 100(1 - T) de cette distribution. Désignant par $\Phi(x)$ la fonction de répartition de la population normale standard située en-deçà de $x$ et par $\phi(x)$ sa densité à $x$, nous avons

$$
c=\Phi^{-1}(1-\mathrm{T}) .
$$

Les quantités $\mathrm{T}_{2}, \mathrm{~T}_{3}$, etc. sont aussi des fractions, $\mathrm{T}_{r}$ étant la fraction de l'hypervolume déterminé dans la distribution multinormale d'ordre $r$ au delà des points $(c, c, \ldots, c)$. Désignons ces fractions supérieures par $h_{r}$ (pour fractions hautes), et les fractions basses complémentaires par $b_{r}$ ou, plus explicitement, $b_{r, \rho}(c)$, ou $\Phi_{r, \rho}(c)$. Nous avons donc

$$
b_{r, \rho}(c)=\operatorname{pr}\left\{\mathrm{X}_{1}<c, \mathrm{X}_{2}<c, \ldots, \mathrm{X}_{r}<c \mid \mathrm{X} \sim N\left(\mathbf{0}_{r}, \mathbf{1}_{r} ; \rho\right)\right\},
$$

en nous référant à la loi multinormale standard, à corrélations homogènes $\rho$ entre les $\mathrm{X}_{j}$. On montre aisément que $h_{1}(c)=1-b_{1}(c)$ où $h_{1}=1-b_{1} ; h_{2}=1-2 b_{1}+b_{2}$, et, en général,

$$
h_{r}=\left(1-b_{u}\right)^{r} \text {, }
$$

l'expression se développant comme un binôme, sauf que la variable $u$, variant de 0 à $r$, apparaît en indice (i.e. $b_{u}$ ) plutôt qu'en exposant $\left(b^{u}\right)$.

Basé sur les équivalences précédentes, le théorème suivant permet de déterminer la fraction supérieure $\mathrm{T}\left(=\mathrm{T}_{1}\right)$, ou encore le seuil $c(=\Phi[1-\mathrm{T}])$, applicable à chacun des $k$ tests, les tests ayant entre eux une intercorrélation commune $\rho$ :

$$
b_{k, \rho}(c)=1-P .
$$

Pour $k=2$ tests, en effet, l'équation (6) peut s'écrire $P=$ $2 h_{1}-h_{2}=2\left(1-b_{1}\right)-\left(1-2 b_{1}+b_{2}\right)=1-b_{2}$, ou bien $b_{2}=1-P$; ainsi de suite pour l'équation (7) et les équations d'ordre supérieur.

Il va sans dire que, une fois $\mathrm{T}\left(=\mathrm{T}_{1}\right)$ déterminé, imposant du même coup le seuil $c$, les fractions d'ordre supérieur, telles $\mathrm{T}_{2}, \mathrm{~T}_{3}$, etc., en découlent directement et sont mesurables par intégration de la surface multinormale au delà des seuils $c$.

La valeur accrue du différentiel et trois stratégies d'attribution. Il existe en fait plusieurs manières d'exploiter la sélection différentielle telle qu'abordée plus haut. Nous distinguons notamment les trois suivantes:

A Un individu est retenu s'il se qualifie au moins dans un test et, s'il se qualifie à plus d'un test, il est assigné au test (c'est-à-dire, à l'emploi) dans lequel son score $\mathrm{X}_{j}$ est maximum. C'est à ce mode de sélection et d'attribution que, à l'instar de BROGDEN (1951), correspond notre concept de différentiel de sélection multiple, $\Delta_{k, \rho}(P)$.

$\mathrm{B}$ Un individu n'est retenu que s'il se qualifie à tous les tests. Ce mode de sélection, plus sévère, assure néanmoins des employés polyvalents, assignables indiffé-

2. Posant $P=2 \mathrm{~T}-\mathrm{T}_{2}$, nous obtenons $1-P=1-2 \mathrm{~T}+\mathrm{T}_{2}=(1-\mathrm{T})^{2}$, d'où la solution suit. 
remment à l'un ou l'autre emploi. Le candidat est attribué au test (et à l'emploi) dans lequel son score est maximum, et le gain escompté correspond à notre concept de différentiel de sélection polyvalent, $\Delta_{k, \rho}^{p}(P)$.

C Un individu n'est retenu que s'il se qualifie à tous les tests et, alors, il est assigné au hasard à l'un des emplois en enjeu. Ce mode d'attribution des individus sélectionnés correspond aussi à un avantage de sélection, mesuré par le différentiel de sélection conjoint, que nous désignerons $\Delta_{k, \rho}^{c}(P)$. Nous ne reviendrons qu'en fin de parcours à ce mode de sélection et attribution, d'ailleurs d'un intérêt mineur.

Nous nous attarderons principalement au concept de différentiel de sélection multiple, correspondant au mode "A" de sélection et d'assignation décrit ci-dessus. Les deux autres modes, d'applications plus circonscrites, seront solutionnés dans la foulée de l'étude sur $\Delta_{k, \rho}(P)$.

Le théorème $d u$ différentiel de sélection multiple, $\Delta_{k, \rho}(P)$. Soit la fraction $\mathrm{T}$ et le seuil $c$, déterminés d'après l'équation (11) en fonction du taux de sélection $P$, du nombre de tests $k$ et de leur intercorrélation commune $\rho$. Les individus qualifiés dans un seul test auront des scores variant de $c$ à $\infty$, dans une distribution normale standard. Supposons que l'espérance (ou moyenne) de ces scores est $m_{1}$ (cette valeur, et celles nommées plus bas, sont traitées dans la section suivante); on a évidemment $m_{1}>c$. Pour les individus qualifiés sur deux tests et assignés au test (et à l'emploi) correspondant à leur score maximal, le gain de sélection qu'ils engendrent est indiqué par la moyenne des maximums de leurs scores $\left(\mathrm{X}_{i}, \mathrm{X}_{j}\right)$, soit $E\left\{\max \left(\mathrm{X}_{i}, \mathrm{X}_{j}\right)\right\}$, valeur que nous désignons $m_{2}$. Il en est de même pour les individus se qualifiant dans 3 tests (avec une moyenne espérée $m_{3}$ ), 4 tests (avec $m_{4}$ ), etc. Notons que ces quantités satisfont l'inégalité $c \leq m_{1} \leq m_{2} \leq m_{3}$ etc. Chaque moyenne $m_{r}$, débordant de plus en plus le seuil $c$ à mesure que $r$ croît, apporte ainsi un gain, une augmentation de "performance" escomptée par rapport à la moyenne de la population de base, soit $\mu=0$ (en valeurs standardisées).

Le différentiel de sélection multiple, qui lui-même est une espérance mathématique (ou une moyenne à travers une multitude d'échantillons), est alors la somme des gains escomptés, pondérés chacun par la fraction escomptée d'individus à chaque palier de qualification.

Prenons l'exemple, amorcé plus haut, de $k=3$ tests. Nous devons d'abord déterminer $\mathrm{T}$ et $c$, par le biais de l'équation (11). Puis, les fractions d'individus 1-qualifiés (i.e. qualifiés seulement dans un test), 2-qualifiés et 3qualifiés étant données, disons $f_{1}, f_{2}$ et $f_{3}$, telles que $f_{1}+f_{2}+$ $f_{3}=P$, nous faisons la somme

$$
\begin{aligned}
\Delta_{3, \rho}(P) & =\left[f_{1} m_{1}+f_{2} m_{2}+f_{3} m_{3}\right] / P \\
& =\left[3\left(\mathrm{~T}-2 \mathrm{~T}_{2}+\mathrm{T}_{3}\right) m_{1}+3\left(\mathrm{~T}_{2}-\mathrm{T}_{3}\right) m_{2}+\mathrm{T}_{3} m_{3}\right] / P .
\end{aligned}
$$

Les calculs requis pour trouver les constantes $\mathrm{T}_{r}$ et $m_{r}$, compte tenu des paramètres de sélection $k, P$ et $\rho$, sont explicités dans la section suivante.

Le théorème suivant donne la formule générale du différentiel de sélection multiple :

$$
\Delta_{k, \rho}(P)=\frac{\left(\begin{array}{l}
k \\
1
\end{array}\right)\left[T_{1+u}(1-1)^{k-1}\right] m_{1}(c)+\left(\begin{array}{l}
k \\
2
\end{array}\right)\left[T_{2+u}(1-1)^{k-2}\right] m_{2}(c)+\cdots+\left(\begin{array}{l}
k \\
k
\end{array}\right) T_{k} m_{k}(c)}{P} ;
$$

la formule, pour être applicable, doit être développée symboliquement, en faisant l'expansion du binôme (1 1) ${ }^{k-r}$ conjointement avec les constantes $T_{r+u}$ qui l'accompagnent. L'Appendice présente une preuve heuristique de ce théorème.

Reprenons, pour illustrer le théorème (13), l'exemple de $k=3$ tests. Le théorème peut alors se récrire :

$$
\begin{aligned}
\Delta_{3, \rho}(P) & =\left\{3\left[\mathrm{~T}_{1+u}(1-1)^{2} m_{1}\right]+3\left[\mathrm{~T}_{2+u}(1-1)^{1} m_{2}+1 \mathrm{~T}_{3} m_{3}\right\} / P\right. \\
& =\left\{3\left[\mathrm{~T}_{1+u}(1-2+1)\right] m_{1}+3\left[\mathrm{~T}_{2+u}(1-1)\right] m_{2}+\mathrm{T}_{3} m_{3}\right\} / P \\
& =\left\{3\left[\mathrm{~T}_{1}-2 \mathrm{~T}_{2}+\mathrm{T}_{3}\right] m_{1}+3\left[\mathrm{~T}_{2}-\mathrm{T}_{3}\right] m_{2}+\mathrm{T}_{3} m_{3}\right\} / P .
\end{aligned}
$$

Les formules des différentiels de sélection multiples pour les cas de $k=1$ à $k=5$ sont données explicitement au tableau 1 .

\section{Méthodes d'évaluation numérique}

Le modèle heuristique que nous proposons pour concrétiser numériquement les différentiels de sélection est celui de la distribution normale, plus spécifiquement la distribution multinormale homogène. Nous identifions d'abord la fonction de densité de cette distribution et proposons ensuite une méthode d'intégration permettant de calculer les constantes $b_{r, \rho}(c)$ et $h_{r, \rho}(c) \quad\left(=\mathrm{T}_{r}\right)$ requises. Puis, nous nous ingénions à déterminer les quantités $m_{r}$, les espérances du maximum de $r$ variables normales homogènes bornées (au delà de $c$ ), par intégration numérique 
Tableau 1 - Formules algébriques des différentiels de sélection multiples pour $k=1$ à 5

\begin{tabular}{lc}
\hline $\mathrm{k}$ & $\Delta_{k, \rho}(P)\{$ chaque expression doit être divisée par $P\}$ \\
\hline 1 & $\mathrm{~T}_{1} m_{1}$ \\
2 & $2\left[\mathrm{~T}_{1}-\mathrm{T}_{2}\right] m_{1}+\mathrm{T}_{2} m_{2}$ \\
3 & $3\left[\mathrm{~T}_{1}-2 \mathrm{~T}_{2}+\mathrm{T}_{3}\right] m_{1}+3\left[\mathrm{~T}_{2}-\mathrm{T}_{3}\right] m_{2}+\mathrm{T}_{3} m_{3}$ \\
4 & $4\left[\mathrm{~T}_{1}-3 \mathrm{~T}_{2}+3 \mathrm{~T}_{3}-\mathrm{T}_{4}\right] m_{1}+6\left[\mathrm{~T}_{2}-2 \mathrm{~T}_{3}+\mathrm{T}_{4}\right] m_{2}+4\left[\mathrm{~T}_{3}-\mathrm{T}_{4}\right] m_{3}+\mathrm{T}_{4} m_{4}$ \\
5 & $5\left[\mathrm{~T}_{1}-4 \mathrm{~T}_{2}+6 \mathrm{~T}_{3}-4 \mathrm{~T}_{4}+\mathrm{T}_{5}\right] m_{1}+10\left[\mathrm{~T}_{2}-3 \mathrm{~T}_{3}+3 \mathrm{~T}_{4}-\mathrm{T}_{5}\right] m_{2}+10\left[\mathrm{~T}_{3}-2 \mathrm{~T}_{4}+\mathrm{T}_{5}\right] m_{3}+5\left[\mathrm{~T}_{4}-\mathrm{T}_{5}\right] m_{4}+\mathrm{T}_{5} m_{5}$ \\
\hline
\end{tabular}

puis par estimation Monte Carlo.

La loi multinormale homogène. Par distribution multinormale homogène, nous entendons ici la distribution conjointe de variables standardisées (d'espérances 0 et de variances 1) ayant deux à deux une corrélation commune $\rho$ La loi de densité multinormale standard étant :

$$
f\left(\mathbf{x}_{1}, \mathbf{x}_{2}, \ldots, \mathbf{x}_{k}\right)=\frac{1}{(2 \pi)^{k / 2} \sqrt{|\mathbf{R}|}} \exp \left[-0,5 \mathbf{x}^{\prime} \mathbf{R}^{-1} \mathbf{x}\right]
$$

avec $\mathbf{x}^{\prime}=x_{1}, x_{2}, \ldots, x_{k}$ dénotant le vecteur (transposé) des observations, la matrice de corrélations (ou covariances) $\mathbf{R}$ et son inverse $\mathbf{R}^{-1}$ sont ici :

$$
\mathbf{R}=\left[\begin{array}{cccc}
1 & \rho & \cdots & \rho \\
\rho & 1 & \cdots & \rho \\
\cdots & \cdots & \cdots & \cdots \\
\rho & \rho & \cdots & 1
\end{array}\right]
$$$$
\mathbf{R}^{-1}=\frac{1}{(1-\rho)[1+(k-1) \rho]}\left[\begin{array}{cccc}
1+(k-2) \rho & -\rho & \cdots & -\rho \\
-\rho & 1+(k-2) \rho & \cdots & -\rho \\
\cdots & \cdots & \cdots & \cdots \\
-\rho & -\rho & \cdots & 1+(k-2) \rho
\end{array}\right]
$$

des logiciels supportent cette fonction. De plus, pour des corrélations tendant vers 1 ou vers 0 , nous avons, respectivement, $b_{k, 1}(c) \rightarrow b_{1}(c)$ et $b_{k, 0}(c) \rightarrow\left[b_{1}(c)\right]^{k}$. Les aires opposées $h_{k, \rho}(c)$ peuvent s'obtenir en appliquant la relation (10); notons tout de même $h_{k, 1}(c)=h_{1}(c)$ et $h_{k, 0}(c)=$ $\left[h_{1}(c)\right]^{k}$. Enfin, nous avons $b_{k, 2}(0)=h_{k, 2}(0)=1 /(k+1)$.

Le cas de la loi binormale (avec $k=2$ ) a été abondamment étudié, et plusieurs procédés d'intégration ont été avancés, la plupart par expansion en série (voir Johnson et coll. 2000). Les méthodes du calcul exact ou approximatif de la corrélation tétrachorique (KENDALL \& STUART, 1979; CASTELLAN, 1966) et de son inversion sont à porter à ce dossier. Notons pour curiosité $b_{2, \rho}(0)=h_{2, \rho}(0)=$ $3+\left(\sin ^{-1} \rho\right) /(2 \pi)$. Notons en passant, pour la loi trinormale $(k=3)$, que $b_{3, \rho}(0)=h_{3, \rho}(0)=1 / 2-3\left(\cos ^{-1} \rho\right) /(4 \pi)$.

Le procédé général qui nous semble le plus commode consiste à utiliser l'expression

De plus, la matrice $\mathbf{R}$ a pour déterminant $|\mathbf{R}|=(1-\rho)^{k-1}[1+$ $(k-1) \rho$ ]. L'incorporation de ces quantités dans (15) nous permet de proposer, selon une formule explicite pour tout $k$, la loi (ou fonction de densité) de la distribution multinormale homogène, soit :

$$
\begin{aligned}
f\left(x_{1}, x_{2}, \ldots, x_{k}\right) & =\frac{1}{(2 \pi)^{k / 2} \sqrt{(1-\rho)^{k-1}[1+(k-1) \rho]}} \\
& \times \exp \left[\frac{[1+(k-2) \rho] \sum_{i} x_{i}^{2}-2 \rho \sum_{i} \sum_{i<j} x_{i} x_{j}}{-2(1-\rho)[1+(k-1) \rho]}\right] .
\end{aligned}
$$

Intégration et détermination des constantes $b_{k, \rho}(c)$ et $h_{k, \rho}(c)$. Pour simplifier la présentation, nous employons la notation $b_{k, \rho}(c)$ définie en (9), plutôt que $\Phi_{k, \rho}(c)$, pour désigner l'aire de l'hyper-volume de $\{-\infty,-\infty, \ldots,-\infty\}$ à $\{c, c, \ldots, c\}$ sous la distribution multinormale.

Certains cas particuliers très simples valent d'être mentionnés. D'abord, nous considérons la fonction $b_{1}(c)=$ $\Phi(c)=\operatorname{Pr}\{\mathrm{X}<c\}$ comme étant élémentaire : la plupart en procédant par intégration numérique. La fonction à intégrer dans (18), disons $w(x)$, a pour mode $\hat{x}$ tel que $w(\hat{x})=$ 0 , où :

$$
w^{\prime}(x)=x \cdot \Phi\left(\frac{c-\sqrt{\rho} \cdot x}{\sqrt{1-\rho}}\right)+k \sqrt{\frac{\rho}{1-\rho}} \cdot \phi\left(\frac{c-\sqrt{\rho} \cdot x}{\sqrt{1-\rho}}\right) .
$$

Ce mode est évidemment de 0 si $\rho=0$, puisqu'alors, le terme de droite dans (19) s'annule, sinon $\hat{x}<0$. Le mode tend vers 0 si $c \rightarrow \infty$, et il tend à suivre $c$ vers la négative (i.e. $\hat{x} \rightarrow c$ si $c<0$ ). L'intégration numérique a avantage à être effectuée séparément, dans chaque intervalle semi-ouvert de part et d'autre du mode $\hat{x}$.

Calcul des maximums $\left(m_{r}\right)$ par intégration numérique. Les quantités $m_{r}$, dénotant la valeur moyenne attendue chez les individus sélectionnés, représentent ainsi l'avantage de sélection. Ces quantités, notées plus explicitement $m_{r, \rho}(c)$, sont utilisées comme constantes dans les différentiels de sélection et elles dénotent l'espérance du maximum de $r$ variables multinormales homogènes, à corrélation $\rho$, bornées chacune dans l'intervalle semi-ouvert $[c, \infty)$ : on peut parler aussi des maximums supnormaux d'ordre $r$. 
Pour $r=1$, ou $m_{1}$, nous cherchons simplement l'espérance d'une variable $\mathrm{X}$ normale dans l'intervalle $[c, \infty)$, d'aire $h_{1}=1-\Phi(c)$. Or, les moments de X sont obtenus simplement, en remarquant que les dérivées de la densité normale $\phi(\mathrm{X})$ font apparaître en facteurs les différentes puissances de $X .{ }^{3}$ Nous obtenons ainsi

$$
\begin{aligned}
E\{\mathrm{X} \mid \mathrm{X}>c\} & =m_{1}=\phi(c) / h_{1}(c) \\
\operatorname{var}\{\mathrm{X} \mid \mathrm{X}>c\} & =1-m_{1}\left(m_{1}-c\right) .
\end{aligned}
$$

Notons aussi comme cas spéciaux importants $m_{r, 1}(c)=$ $m_{1}(c)$ et $m_{2, \rho}(-\infty)=\sqrt{(1-\rho) / \pi}$.

La densité du maximum $y$ de $r$ variables à densité conjointe $f\left(x_{1}, x_{2}, \ldots, x_{r}\right)$ est :

$g_{r}(y)=k \cdot \int_{c}^{y} \int_{c}^{y} \cdots \int_{c}^{y} f\left(y, x_{2}, \cdots, x_{k}\right) d x_{k} d x_{k-1} \cdots d x_{2} / h_{k, \rho}(c)$;

cette fonction de densité va de $c$ à $\infty$ et n'est pas réductible, sauf lorsque les variables $\mathrm{X}_{j}$ sont mutuellement indépendantes, avec $\rho=0$. Nous considérons ce cas plus loin. Si on dispose de la densité (21), l'espérance cherchée est alors, simplement,

$$
m_{r, \rho}(c)=\int_{C}^{\infty} y \cdot g_{r}(y) d y
$$

Pour $k=2$, la densité est très simple et, après quelques manipulations, nous obtenons :

$$
g_{2}(y)=2 \phi(y)\left[\Phi\left(\frac{(\rho \cdot y-c)}{\sqrt{1-\rho^{2}}}\right)+\Phi\left(y \sqrt{\frac{1-\rho}{1+\rho}}\right)-1\right] \div \mathrm{h}_{2, \rho}(c)
$$

expression qui, avec l'expression (22), nous permet la détermination précise de $m_{2}$.

Pour $k=3$, le procédé mis en oeuvre pour $k=2$ peut être prolongé d'un ordre, en trouvant d'abord :

$$
\begin{aligned}
t\left(y, x_{1}\right)= & \int_{c}^{y} f\left(y, x_{1}, x_{2}\right) d x_{2} \\
= & \left\{\Phi\left[\frac{y-\rho \cdot x_{1}}{\sqrt{\left(1-\rho^{2}\right)(1+2 \rho)}}\right]-\Phi\left[\frac{c(1+\rho)-\rho\left(y+x_{1}\right)}{\sqrt{\left(1-\rho^{2}\right)(1+2 \rho)}}\right]\right\} \\
& \times \frac{\exp \left[-\frac{y^{2}-2 \rho x_{1} y+x_{1}^{2}}{2\left(1-\rho^{2}\right)}\right]}{2 \pi \sqrt{\frac{\left.\left(1-3 \rho^{2}+2 \rho^{3}\right)(1+\rho)\right)}{(1-\rho)(1+2 \rho)}}} .
\end{aligned}
$$

La densité $g_{3}(y)$ peut alors être calculée approximativement par intégration numérique de $t\left(y, x_{1}\right)$ dans l'intervalle $[c, y]$ pour finalement être incorporée dans (22) afin de déterminer $m_{3}$.

Les quantités $m_{r, 0}(c)$, à corrélations 0 , constituent un cas à part puisqu'alors, les variables $\mathrm{X}_{j}$ étant indépendantes, leur densité conjointe (17) peut être factorisée de même que l'expression de leur intégrale multiple.

Soit $\Phi(\mathrm{X})$ et $\phi(\mathrm{X})$, les fonctions de répartition et de densité de la loi normale standard. Restreignant $\mathrm{X}$ dans l'intervalle $[c, \infty]$, la densité de cette variable restreinte est évidemment $\phi(\mathrm{X}) /(1-P)$, où $P=\Phi(c)$, et l'intégrale correspondante, $F(\mathrm{X})=[\Phi(\mathrm{X})-P] /(1-P)$.

Maintenant, utilisant $r$ variables semblables et indépendantes, la probabilité que leur maximum soit $y$ est la même que la probabilité qu'elles soient toutes conjointement inférieures à $y$, soit

$$
G_{r}(y)=G\left[\max \left(x_{1}, x_{2}, \ldots, x_{r}\right)=y\right]=\{F(y)\}^{r} .
$$

La fonction de répartition (du maximum) peut être appliquée directement pour trouver l'espérance $\mathrm{E}(\mathrm{y})$, correspondant ici à $m_{r}, 0(\mathbf{c})$. Le calcul à opérer est :

$$
E(y)=c+\int\left[1-G_{r}(y)\right] \mathrm{d} y \quad\{y \geq c\}
$$

les bornes d'intégration sont ici $c$ et $\infty$. Une autre façon consiste à dériver (25) pour obtenir la densité $g_{r}$ du maximum de $r$ variables normales indépendantes, bornées inférieurement par $c$, soit :

$$
g_{r}(y)=r\left[\frac{\Phi(y)-P}{1-P}\right]^{r-1} \cdot \frac{\phi(y)}{1-P},
$$

grâce à laquelle tous calculs souhaitables peuvent ensuite être effectués.

Pour des volumes de dimension plus élevée ( $r \geq 4)$, l'intégration numérique directe de (21) reste possible, grâce à la puissance des ordinateurs et à l'efficacité de la programmation mise en oeuvre. C'est ainsi, par intégration récursive sur $r$, que nous avons obtenu les maximums $m_{r}$ exploités dans le calcul des différentiels présentés, garantissant un résultat précis à \pm 0.0001 .

Calcul des maximums $\left(m_{r}\right)$ par simulations Monte Carlo. Si et lorsque les méthodes présentées dans les paragraphes précédents ne permettent pas d'obtenir les valeurs $m_{r, \rho}(c)$ cherchées, l'estimation Monte Carlo reste une autre voie possible.

Le procédé d'estimation par la méthode Monte Carlo (LAURENCELLE, 2001) consiste essentiellement (1) à produire des valeurs possibles de la variable étudiée à partir d'une manipulation adéquate des nombres aléatoires, et (2) à calculer les estimés-synthèses requis, tels la moyenne et les autres moments de la distribution empirique. Dans le cas de $y=\max \left(x_{1}, x_{2}, \ldots, x_{r}\right)$ et de son espérance $m_{r, \rho}(c)$, il

\footnotetext{
3. Rappelant $\phi(\mathrm{X})=\exp \left(-\mathrm{X}^{2} / 2\right) / \sqrt{2 \pi}$, nous avons $\mathrm{d} \phi(\mathrm{X}) / \mathrm{dX}=-\mathrm{X} \phi(\mathrm{X})$ et $\mathrm{d}^{2} \phi(\mathrm{X}) /(\mathrm{dX})^{2}=\left(\mathrm{X}^{2}-1\right) \phi(\mathrm{X})$.
} 
suffit de produire un grand échantillon de valeurs statistiquement adéquates, i.e. $\left\{y_{1}, y_{2}, \ldots, y_{N}\right\}$, puis d'obtenir

$$
\hat{m}_{r \rho}=\operatorname{moy}\left[y_{1}, y_{2}, \ldots, y_{N}\right] .
$$

Cet estimateur simple est sans biais et sa précision, reflétée par sa variance, $\operatorname{var}\left(\hat{m}_{r, \rho}\right)=\sigma_{y}^{2} / N$, dépend de $N$ : la précision est en fait inversement proportionnelle à $\sqrt{N}$

Deux techniques de production de $y$, pour des conditions générales de $c$ (le seuil), $r$ (le nombre de variables) et $\rho$ (leur intercorrélation commune), se présentent au regard.

Production symétrique de variables normales corrélées. La méthode symétrique produit un vecteur $\left\{x_{1}, x_{2}, \ldots, x_{r}\right\}$ à partir de $r+1$ variables aléatoires normales indépendantes standard $\left\{z_{0}, z_{1}, z_{2}, \ldots, z_{r}\right\}$, chaque $\mathrm{X}_{j}$ étant obtenu selon

$$
x_{j}=\sqrt{\rho} z_{0}+\sqrt{1-\rho} z_{j} .
$$

Le lecteur pourra vérifier lui-même les propriétés idoines de cette transformation. Il reste à établir l'algorithme de détermination du maximum $y$ et le calcul de la moyenne des estimés. La méthode la plus courante pour produire des variables normales standard est la méthode dite de BoxMuller, soit $z \leftarrow \sqrt{-2 \log _{e} u_{1}} \times \sin \left(2 \pi u_{2}\right)$, où $u_{1}$ et $u_{2}$ sont des variables aléatoires uniformes indépendantes, dans l'intervalle $[0,1)$.

L'algorithme symétrique pour l'estimation de $m_{r, \rho}$ peut être construit selon les étapes suivantes de l'algorithme 1 (voir plus loin). À l'étape 0, une façon directe d'obtenir $z_{0}$ dans l'intervalle $[c, \infty)$ consiste à produire d'abord une variable uniforme $w$ (disons) dans l'intervalle $[\Phi(c), 1)$, par $w \leftarrow \Phi(c)+[1-\Phi(c)] u, u$ étant aléatoire et uniforme dans $[0,1)$, puis à inverser l'intégrale normale, soit $z_{0} \leftarrow$ $\Phi^{-1}(w)$. Johnson, Kotz et BALAKRishnan (1994) et DEVROYE (1986) documentent les techniques et algorithmes d'inversion.

Production cumulative de variables normales corrélées. La méthode cumulative produit un vecteur $\left\{x_{1}, x_{2}, \ldots, x_{r}\right\}$ à partir d'un nombre égal $(r)$ de variables aléatoires normales indépendantes standard $\left\{z_{1}, z_{2}, \ldots, z_{r}\right\}$ et d'un jeu de constantes. Chaque $\mathrm{X}_{j}$ est déterminé alors par :

$$
\begin{aligned}
x_{j} & =\sum_{i=1}^{j} \beta_{j, i} z_{j} \\
& =\sum_{i=1}^{j-1} \frac{\rho(1-\rho)}{\sqrt{(1-\rho)(1+[i-2] \rho)(1+[i-1] \rho)}} \times z_{i} \\
& +\sqrt{1-\frac{(j-1) \rho^{2}}{1+(j-2) \rho}} \times z_{j} .
\end{aligned}
$$

Ces constantes $\beta_{j, i}$ peuvent naturellement être fixées d'avance. Les premières transformations sont $x_{1}=z_{1} ; x_{2}=$ $\rho z_{1}+\sqrt{1-\rho^{2}} z_{2}$; etc. Le schéma séquentiel approprié est donné à l'Algorithme 2. L'algorithme cumulatif est ainsi nommé parce que chaque nouvelle variable $\mathrm{X}_{j}$ rajoute une variable $z_{j}$ supplémentaire.

L'étude, mathématique et empirique des deux méthodes montre clairement que, malgré ses constantes multiplicatives d'apparence plus compliquée, la méthode cumulative est plus performante. Elle trouve plus efficacement et plus rapidement les maximums $y$ dans l'hypervolume multinormal que ne le fait la méthode symétrique.

Cependant, l'intégration numérique est encore possible pour évaluer plus précisément les moments (surtout l'espérance) du maximum de $r$ variables supnormales, ce en évaluant (21) par une récursion sur $r$, la densité étant fournie par (17). Les valeurs présentées, notamment aux tableaux 6 à 9, ont été ainsi calculées.

\section{Valeurs des différentiels de sélection multiples, polyva- lents et conjoints}

La valeur des différentiels de sélection, selon qu'ils soient multiples, polyvalents ou conjoints, peut être calculée au besoin, pour satisfaire un contexte particulier, en employant les méthodes et procédures proposées ici. Dans le cas des deux premiers types de différentiels, nous avons cependant cru qu'il pût être intéressant de fournir des tables de valeurs calculées à la fois pour illustrer les concepts, servir de base d'interpolation pour des applications particulières ou servir de repère pour qui voudrait reprendre les calculs.

Valeurs des différentiels de sélection multiples. Les tableaux 2 à 5 présentent les valeurs calculées des seuils $c$ et des différentiels de sélection multiples, $\Delta_{k, \rho}(P)$, applicables selon le nombre de couples test :emploi considérés $(1 \leq k \leq 5)$, le taux de sélection $(P=0,1,0,05$ et 0,01$)$ et la corrélation entre tests $(\rho=0,0(0,1) 0,9 ; 0,95 ; 1)$. Dans un premier temps, nous avons utilisé le théorème (11) afin de déterminer le seuil $c$ et le taux individuel $\mathrm{T}$ correspondant. Puis nous avons mis en oeuvre l'évaluation du théorème (13), en trouvant les fractions $\mathrm{T}_{r}$ appropriées selon l'intégrale (18), et les maximums $m_{r}$, soit par intégration (cf. éq. 20-27), soit par estimation Monte Carlo (cf. éq. 28-30. Les quantités ont été établies généralement à 5 chiffres de précision, sauf pour les combinaisons $k=4$ et $k=5,0<\rho<1$, pour lesquelles l'estimation Monte Carlo nous contraignait à de plus modestes ambitions. Les nombres rapportés sont donc précis tels quels, à la dernière décimale près.

Rappelons que, en vertu des équations (3-5), le gain (moyen) escompté par la sélection dans l'emploi $j$ est indiqué par

$$
\overline{\mathrm{Y}}_{s}-\mu_{y}=\rho\left(\mathrm{X}_{j}, \mathrm{Y}_{j}\right) \sigma\left(\mathrm{Y}_{j}\right) \Delta_{k, \rho}(P) .
$$

Valeurs des différentiels de sélection polyvalents. Les différentiels de sélection polyvalents sont, en valeurs standardi- 
Tableau 2 घ Différentiels de sélection d'ordre 2, $\Delta_{2, \rho}(P)$

\begin{tabular}{|c|c|c|c|c|c|c|c|c|}
\hline \multirow[b]{2}{*}{$\rho$} & \multicolumn{2}{|c|}{$P=0,50$} & \multicolumn{2}{|c|}{$P=0,10$} & \multicolumn{2}{|c|}{$P=0,05$} & \multicolumn{2}{|c|}{$P=0,01$} \\
\hline & $c$ & $\Delta_{2, \rho}$ & $c$ & $\Delta_{2, \rho}$ & $c$ & $\Delta_{2, \rho}$ & $c$ & $\Delta_{2, \rho}$ \\
\hline 1 & 0 & 0,7979 & 1,2816 & 1,7550 & 1,6449 & 2,0627 & 2,3263 & 2,6652 \\
\hline ,95 & 0,1260 & 0,9812 & 1,3796 & 1,9728 & 1,7581 & 2,2717 & 2,4346 & 2,8563 \\
\hline, 9 & 0,1780 & 1,0948 & 1,4397 & 2,0131 & 1,7976 & 2,3055 & 2,4694 & 2,8796 \\
\hline ,8 & 0,2511 & 1,1593 & 1,4931 & 2,0418 & 1,8460 & 2,3269 & 2,5091 & 2,8907 \\
\hline, 7 & 0,3067 & 1,1899 & 1,5292 & 2,0504 & 1,8773 & 2,3320 & 2,5324 & 2,8917 \\
\hline ,6 & 0,3531 & 1,2056 & 1,5560 & 2,0527 & 1,8997 & 2,3328 & 2,5476 & 2,8912 \\
\hline, 5 & 0,3935 & 1,2134 & 1,5770 & 2,0532 & 1,9163 & 2,3327 & 2,5578 & 2,8906 \\
\hline ,4 & 0,4296 & 1,2170 & 1,5936 & 2,0533 & 1,8289 & 2,3326 & 2,5647 & 2,8905 \\
\hline ,3 & 0,4623 & 1,2185 & 1,6069 & 2,0537 & 1,9385 & 2,3330 & 2,5692 & 2,8906 \\
\hline, 2 & 0,4922 & 1,2192 & 1,6175 & 2,0545 & 1,9456 & 2,3335 & 2,5722 & 2,8909 \\
\hline ,1 & 0,5196 & 1,2200 & 1,6258 & 2,0556 & 1,9508 & 2,3343 & 2,5739 & 2,8912 \\
\hline, 0 & 0,5450 & 1,2215 & 1,6322 & 2,0570 & 1,9545 & 2,3353 & 2,5750 & 2,8916 \\
\hline
\end{tabular}

Tableau 3 ■ Différentiels de sélection d'ordre 3, $\Delta_{3, \rho}(P)$

\begin{tabular}{|c|c|c|c|c|c|c|c|c|}
\hline \multirow[b]{2}{*}{$\rho$} & \multicolumn{2}{|c|}{$P=0,50$} & \multicolumn{2}{|c|}{$P=0,10$} & \multicolumn{2}{|c|}{$P=0,05$} & \multicolumn{2}{|c|}{$P=0,01$} \\
\hline & $c$ & $\Delta_{3, \rho}$ & $c$ & $\Delta_{3, \rho}$ & $c$ & $\Delta_{3, \rho}$ & $c$ & $\Delta_{3, \rho}$ \\
\hline 1 & 0 & 0,7979 & 1,2816 & 1,7550 & 1,6449 & 2,0627 & 2,3263 & 2,6652 \\
\hline ,95 & 0,1891 & 1,1539 & 1,4567 & 2,0887 & 1,8162 & 2,3856 & 2,4906 & 2,9636 \\
\hline, 9 & 0,2671 & 1,2563 & 1,5209 & 2,1553 & 1,8767 & 2,4412 & 2,5444 & 3,0019 \\
\hline ,8 & 0,3770 & 1,3587 & 1,6031 & 2,2002 & 1,9516 & 2,4773 & 2,6068 & 3,0173 \\
\hline, 7 & 0,4607 & 1,4062 & 1,6589 & 2,2135 & 2,0005 & 2,4818 & 2,6440 & 3,0188 \\
\hline, 6 & 0,5307 & 1,4285 & 1,7008 & 2,2163 & 2,0358 & 2,4813 & 2,6684 & 3,0174 \\
\hline, 5 & 0,5917 & 1,4230 & 1,7335 & 2,2157 & 2,0621 & 2,4824 & 2,6849 & 3,0157 \\
\hline, 4 & 0,6462 & 1,4400 & 1,7595 & 2,2052 & 2,0820 & 2,4801 & 2,6959 & 3,0163 \\
\hline ,3 & 0,6955 & 1,4396 & 1,7801 & 2,2151 & 2,0969 & 2,4804 & 2,7032 & 3,0161 \\
\hline, 2 & 0,7405 & 1,4384 & 1,7964 & 2,2162 & 2,1080 & 2,4811 & 2,7078 & 3,0162 \\
\hline, 1 & 0,7817 & 1,4382 & 1,8089 & 2,2177 & 2,1158 & 2,4828 & 2,7105 & 3,0165 \\
\hline, 0 & 0,8193 & 1,4404 & 1,8183 & 2,2198 & 2,1212 & 2,4840 & 2,7119 & 3,0176 \\
\hline
\end{tabular}

Tableau 4 ■ Différentiels de sélection d'ordre $4, \Delta_{4, \rho}(P)$

\begin{tabular}{|c|c|c|c|c|c|c|c|c|}
\hline \multirow[b]{2}{*}{$\rho$} & \multicolumn{2}{|c|}{$P=0,50$} & \multicolumn{2}{|c|}{$P=0,10$} & \multicolumn{2}{|c|}{$P=0,05$} & \multicolumn{2}{|c|}{$P=0,01$} \\
\hline & $c$ & $\Delta_{4, \rho}$ & $c$ & $\Delta_{4, \rho}$ & $c$ & $\Delta_{4, \rho}$ & $c$ & $\Delta_{4, \rho}$ \\
\hline 1 & 0 & 0,7979 & 1,2816 & 1,7550 & 1,6449 & 2,0627 & 2,3263 & 2,6652 \\
\hline ,95 & 0,2300 & 1,2362 & 1,4954 & 2,1730 & 1,8543 & 2,4574 & 2,5276 & 3,0290 \\
\hline, 9 & 0,3250 & 1,3651 & 1,5744 & 2,2507 & 1,9289 & 2,5217 & 2,5943 & 3,0749 \\
\hline ,8 & 0,4588 & 1,4940 & 1,6759 & 2,3133 & 2,0219 & 2,5764 & 2,6724 & 3,0974 \\
\hline, 7 & 0,5609 & 1,5534 & 1,7452 & 2,3249 & 2,0830 & 2,5846 & 2,7194 & 3,1073 \\
\hline ,6 & 0,6462 & 1,5802 & 1,7974 & 2,3290 & 2,1272 & 2,5833 & 2,7505 & 3,1053 \\
\hline, 5 & 0,7207 & 1,5891 & 1,8383 & 2,3269 & 2,1603 & 2,5824 & 2,7716 & 3,1024 \\
\hline, 4 & 0,7873 & 1,5897 & 1,8707 & 2,3242 & 2,1854 & 2,5806 & 2,7857 & 3,1025 \\
\hline ,3 & 0,8475 & 1,5870 & 1,8965 & 2,3246 & 2,2042 & 2,5818 & 2,7950 & 3,1022 \\
\hline ,2 & 0,9024 & 1,5839 & 1,9167 & 2,3250 & 2,2180 & 2,5818 & 2,8008 & 3,1023 \\
\hline, 1 & 0,9526 & 1,5911 & 1,9320 & 2,3273 & 2,2276 & 2,5838 & 2,8041 & 3,1032 \\
\hline, 0 & 0,9981 & 1,5862 & 1,9432 & 2,3300 & 2,2340 & 2,5859 & 2,8058 & 3,1040 \\
\hline
\end{tabular}


Tableau 5 - Différentiels de sélection d'ordre $5, \Delta_{5, \rho}(P)$

\begin{tabular}{|c|c|c|c|c|c|c|c|c|}
\hline \multirow[b]{2}{*}{$\rho$} & \multicolumn{2}{|c|}{$P=0,50$} & \multicolumn{2}{|c|}{$P=0,10$} & \multicolumn{2}{|c|}{$P=0,05$} & \multicolumn{2}{|c|}{$P=0,01$} \\
\hline & $c$ & $\Delta_{5, \rho}$ & $c$ & $\Delta_{5, \rho}$ & $c$ & $\Delta_{5, \rho}$ & $c$ & $\Delta_{5, \rho}$ \\
\hline 1 & 0 & 0,7979 & 1,2816 & 1,7550 & 1,6449 & 2,0627 & 2,3263 & 2,6652 \\
\hline ,95 & 0,2599 & 1,2978 & 1,5239 & 2,2281 & 1,8823 & 2,5168 & 2,5548 & 3,0880 \\
\hline ,9 & 0,3673 & 1,4462 & 1,6137 & 2,3256 & 1,9674 & 2,5998 & 2,6313 & 3,1467 \\
\hline 8 & 0,5186 & 1,5958 & 1,7296 & 2,3909 & 2,0740 & 2,5909 & 2,7213 & 3,1592 \\
\hline, 7 & 0,6340 & 1,6640 & 1,8091 & 2,4102 & 2,1443 & 2,6659 & 2,7759 & 3,1718 \\
\hline ,6 & 0,7307 & 1,6942 & 1,8691 & 2,4126 & 2,1954 & 2,6548 & 2,8123 & 3,1716 \\
\hline ,5 & 0,8150 & 1,7033 & 1,9162 & 2,4093 & 2,2338 & 2,6599 & 2,8370 & 3,1682 \\
\hline ,4 & 0,8904 & 1,7023 & 1,9537 & 2,4082 & 2,2629 & 2,6566 & 2,8536 & 3,1679 \\
\hline ,3 & 0,9587 & 1,6965 & 1,9834 & 2,4070 & 2,2847 & 2,6569 & 2,8645 & 3,1675 \\
\hline ,2 & 1,0210 & 1,6917 & 2,0065 & 2,4085 & 2,3006 & 2,6575 & 2,8712 & 3,1673 \\
\hline , 1 & 1,0777 & 1,6898 & 2,0240 & 2,4098 & 2,3116 & 2,6607 & 2,8750 & 3,1685 \\
\hline ,0 & 1,1290 & 1,6932 & 2,0365 & 2,4127 & 2,3187 & 2,6616 & 2,8769 & 3,1694 \\
\hline
\end{tabular}

Tableau 6 - Différentiels de sélection polyvalents d'ordre 2, $\Delta_{2, \rho}(P)$

\begin{tabular}{|c|c|c|c|c|c|c|c|c|}
\hline \multirow[b]{2}{*}{$\rho$} & \multicolumn{2}{|c|}{$P=0,50$} & \multicolumn{2}{|c|}{$P=0,10$} & \multicolumn{2}{|c|}{$P=0,05$} & \multicolumn{2}{|c|}{$P=0,01$} \\
\hline & $c$ & $\Delta_{2, \rho}^{p}$ & $c$ & $\Delta_{2, \rho}^{p}$ & $c$ & $\Delta_{2, \rho}^{p}$ & $c$ & $\Delta_{2, \rho}^{p}$ \\
\hline 1 & 0 & 0,7979 & 1,2816 & 1,7550 & 1,6449 & 2,0627 & 2,3263 & 2,6652 \\
\hline 95 & $-0,1260$ & 0,9031 & 1,1451 & 1,8366 & 1,5053 & 2,1370 & 2,1810 & 2,7255 \\
\hline ,9 & $-0,1780$ & 0,9341 & 1,0823 & 1,8450 & 1,4393 & 2,1385 & 2,1088 & 2,7142 \\
\hline 8 & $-0,2511$ & 0,9647 & 0,9869 & 1,8317 & 1,3372 & 2,1124 & 1,9936 & 2,6641 \\
\hline, 7 & $-0,3067$ & 0,9770 & 0,9081 & 1,8012 & 1,2513 & 2,0695 & 1,8938 & 2,5985 \\
\hline ,6 & $-0,3531$ & 0,9792 & 0,8376 & 1,7611 & 1,1733 & 2,0174 & 1,8011 & 2,5245 \\
\hline ,5 & $-0,3935$ & 0,9743 & 0,7721 & 1,7142 & 1,0999 & 1,9588 & 1,7123 & 2,4444 \\
\hline , 4 & $-0,4296$ & 0,9640 & 0,7099 & 1,6619 & 1,0295 & 1,8949 & 1,6256 & 2,3593 \\
\hline , 3 & $-0,4623$ & 0,9489 & 0,6501 & 1,6049 & 0,9610 & 1,8263 & 1,5400 & 2,2697 \\
\hline ,2 & $-0,4922$ & 0,9297 & 0,5919 & 1,5434 & 0,8936 & 1,7532 & 1,4545 & 2,1754 \\
\hline , 1 & $-0,5196$ & 0,9066 & 0,5347 & 1,4775 & 0,8268 & 1,6756 & 1,3686 & 2,0765 \\
\hline 0 & $-0,5450$ & 0,8796 & 0,4783 & 1,4071 & 0,7601 & 1,5934 & 1,2816 & 1,9726 \\
\hline
\end{tabular}

Tableau 7 - Différentiels de sélection polyvalents d'ordre 3, $\Delta_{3, \rho}(P)$

\begin{tabular}{|c|c|c|c|c|c|c|c|c|}
\hline \multirow[b]{2}{*}{$\rho$} & \multicolumn{2}{|c|}{$P=0,50$} & \multicolumn{2}{|c|}{$P=0,10$} & \multicolumn{2}{|c|}{$P=0,05$} & \multicolumn{2}{|c|}{$P=0,01$} \\
\hline & $c$ & $\Delta_{3, \rho}^{p}$ & $c$ & $\Delta_{3, \rho}^{p}$ & $c$ & $\Delta_{3, \rho}^{p}$ & $c$ & $\Delta_{3, \rho}^{p}$ \\
\hline 1 & 0 & 0,7979 & 1,2816 & 1,7550 & 1,6449 & 2,0627 & 2,3263 & 2,6652 \\
\hline ,95 & $-0,1891$ & 0,9624 & 1,0780 & 1,8911 & 1,4371 & 2,1899 & 2,1106 & 2,7752 \\
\hline, 9 & $-0,2671$ & 1,0156 & 0,9850 & 1,9164 & 1,3398 & 2,2067 & 2,0048 & 2,7757 \\
\hline ,8 & $-0,3770$ & 1,0747 & 0,8443 & 1,9201 & 1,1897 & 2,1935 & 1,8370 & 2,7309 \\
\hline, 7 & $-0,4607$ & 1,1059 & 0,7283 & 1,8956 & 1,0639 & 2,1524 & 1,6921 & 2,6586 \\
\hline 6 & $-0,5307$ & 1,1215 & 0,6245 & 1,8545 & 0,9498 & 2,0947 & 1,5577 & 2,5697 \\
\hline, 5 & $-0,5917$ & 1,1260 & 0,5281 & 1,8011 & 0,8423 & 2,0242 & 1,4286 & 2,4677 \\
\hline 4 & $-0,6462$ & 1,1215 & 0,4363 & 1,7367 & 0,7389 & 1,9426 & 1,3022 & 2,3541 \\
\hline ,3 & $-0,6955$ & 1,1090 & 0,3477 & 1,6621 & 0,6378 & 1,8502 & 1,1765 & 2,2288 \\
\hline ,2 & $-0,7405$ & 1,0890 & 0,2611 & 1,5770 & 0,5376 & 1,7464 & 1,0498 & 2,0911 \\
\hline 1 & $-0,7817$ & 1,0614 & 0,1754 & 1,4802 & 0,4375 & 1,6302 & 0,9208 & 1,9394 \\
\hline 0 & $-0,8193$ & 1,0257 & 0,0900 & 1,3700 & 0,3361 & 1,4994 & 0,7877 & 1,7709 \\
\hline
\end{tabular}


Algorithme 1 - Algorithme symétrique pour l'estimation de $m_{r, \rho}$

INITIALISATION : $\Sigma \rightarrow 0$. Pour $i$ variant de 1 à $N$

CYCLE :

Étape 0 : Produire $z_{0}$ dans l'intervalle $[c, \infty]$

Étape 1 : Produire $z_{1}$ et calculer $x_{1}$ selon la transformation (29); si $x_{1}<c$, reprendre à l'étape 0

Étape 2 : Produire $z_{2}$ et calculer $x_{2}$ selon la transformation (29); si $x_{2}<c$, reprendre à l'étape 0

...

Étape $r$ : Produire $z_{r}$ et calculer $x_{r}$ selon la transformation (29); si $x_{r}<c$, reprendre à l'étape 0

Étape $r+1$ : Trouver $y=\max \left(x_{1}, x_{2}, \ldots, x_{r}\right)$ et faire $\Sigma \rightarrow \Sigma+y$

CONCLUSION : Obtenir l'estimé $\hat{m}_{r \rho}=\Sigma / N$, pour une précision d'ordre $1 / \sqrt{N}$.

Tableau 8 a Différentiels de sélection polyvalents d'ordre 4, $\Delta_{4, \rho}(P)$

\begin{tabular}{|c|c|c|c|c|c|c|c|c|}
\hline \multirow[b]{2}{*}{$\rho$} & \multicolumn{2}{|c|}{$P=0,50$} & \multicolumn{2}{|c|}{$P=0,10$} & \multicolumn{2}{|c|}{$P=0,05$} & \multicolumn{2}{|c|}{$P=0,01$} \\
\hline & $c$ & $\Delta_{4, \rho}^{p}$ & $c$ & $\Delta_{4, \rho}^{p}$ & $c$ & $\Delta_{4, \rho}^{p}$ & $c$ & $\Delta_{4, \rho}^{p}$ \\
\hline 1 & 0 & 0,7979 & 1,2816 & 1,7550 & 1,6449 & 2,0627 & 2,3263 & 2,6652 \\
\hline ,95 & $-0,2300$ & 1,0022 & 1,0349 & 1,8007 & 1,3933 & 2,2277 & 2,0657 & 2,8120 \\
\hline, 9 & $-0,3250$ & 1,0712 & 0,9227 & 1,9688 & 1,2761 & 2,2579 & 1,9388 & 2,8246 \\
\hline ,8 & $-0,4588$ & 1,1516 & 0,7532 & 1,9896 & 1,0960 & 2,2604 & 1,7383 & 2,7923 \\
\hline, 7 & $-0,5609$ & 1,1981 & 0,6138 & 1,9754 & 0,9454 & 2,2278 & 1,5658 & 2,7247 \\
\hline ,6 & $-0,6462$ & 1,2256 & 0,4892 & 1,9402 & 0,8088 & 2,1737 & 1,4059 & 2,6351 \\
\hline, 5 & $-0,7207$ & 1,2394 & 0,3734 & 1,8888 & 0,6801 & 2,1028 & 1,2524 & 2,5278 \\
\hline ,4 & $-0,7873$ & 1,2419 & 0,2631 & 1,8231 & 0,5562 & 2,0168 & 1,1017 & 2,4037 \\
\hline ,3 & $-0,8475$ & 1,2344 & 0,1563 & 1,7431 & 0,4347 & 1,9154 & 0,9512 & 2,2624 \\
\hline ,2 & $-0,9024$ & 1,2171 & 0,0516 & 1,6477 & 0,3140 & 1,7972 & 0,7988 & 2,1017 \\
\hline , & $-0,9526$ & 1,1894 & $-0,0524$ & 1,5344 & 0,1923 & 1,6590 & 0,6422 & 1,9177 \\
\hline 0 & $-0,9981$ & 1,1504 & $-0,1569$ & 1,3985 & 0,0681 & 1,4954 & 0,4783 & 1,7033 \\
\hline
\end{tabular}

sées, l'avantage de sélection encouru pour $k$ emplois en ne retenant que les candidats qui sont qualifiés par les $k$ tests à la fois. La procédure de calcul est ici plus simple. D'abord, à partir du taux de sélection $P$ et du nombre $k$ de tests, il s'agit de résoudre pour $c$ l'équation :

$$
h_{k, \rho}(c)=P,
$$

en utilisant au besoin la transposition (10). Le différentiel polyvalent est alors simplement

$$
\Delta_{k, \rho}^{p}(P)=m_{k, \rho}(c)
$$

Les tableaux 6 à 9 énumèrent les valeurs de ces différentiels polyvalents pour les mêmes combinaisons des paramètres $k, P$ et $\rho$. Le gain escompté par la sélection dans l'emploi j s'établit comme ci-haut (cf. éq. 31 ).

Le lecteur remarquera que les seuils $c$ associés aux différentiels polyvalents sont plus bas que ceux des seuils multiples (sauf évidemment pour $\rho=1$, auquel cas ils coïncident). La raison en est que, le critère de polyvalence étant plus exigeant (chaque individu doit se qualifier à tous les tests afin d'être sélectionné), le seuil c doit être abaissé afin de respecter la même valeur $P$ prescrite du taux de sélection. Il eût été possible de faire autrement, en construisant par exemple les tableaux de valeurs 6 à 9 à partir des seuils $c$ pris dans les tableaux correspondants 2 à 5 et en évaluant alors le taux de sélection effectif $P$ (qui eût été beaucoup moindre) et le différentiel polyvalent associé.

Valeurs des différentiels de sélection conjoints. Les différentiels de sélection conjoints, relatifs à la variante " $\mathrm{C}$ " du concept de différentiel que nous avons mentionnée plus haut, indiquent l'avantage de sélection escompté en recrutant les individus co-qualifiés dans tous les tests et en les assignant dans l'un ou l'autre des $k$ emplois disponibles, au hasard. Ce mode de qualification assure la "polyvalence" déjà évoquée pour le cas des différentiels polyvalents ; pour cette raison, les mêmes seuils $c$, retrouvés dans les tableaux 6 à 9, s'appliquent ici aussi. Par contre, l'attribution au hasard des individus dans les emplois, quelles que soient les valeurs particulières des scores de qualification qu'ils obtiennent, a pour conséquence de réduire l'avantage retrouvé dans les différentiels polyvalents. Le gain escompté ici, dénoté $\Delta_{k, \rho}^{c}(P)$ et exprimé en valeurs standardisées, correspond à l'espérance d'une variable $\mathrm{X}_{j}$ de la loi multinormale (17) confinée dans l'hyper-coin délimité par l'hyper- 
Tableau 9 - Différentiels de sélection polyvalents d'ordre 5, $\Delta_{5, \rho}(P)$

\begin{tabular}{|c|c|c|c|c|c|c|c|c|}
\hline \multirow[b]{2}{*}{$\rho$} & \multicolumn{2}{|c|}{$P=0,50$} & \multicolumn{2}{|c|}{$P=0,10$} & \multicolumn{2}{|c|}{$P=0,05$} & \multicolumn{2}{|c|}{$P=0,01$} \\
\hline & $c$ & $\Delta_{5, \rho}^{p}$ & $c$ & $\Delta_{5, \rho}^{p}$ & $c$ & $\Delta_{5, \rho}^{p}$ & $c$ & $\Delta_{5, \rho}^{p}$ \\
\hline 1 & 0 & 0,7979 & 1,2816 & 1,7550 & 1,6449 & 2,0627 & 2,3263 & 2,6652 \\
\hline ,95 & $-0,2599$ & 1,0317 & 1,0036 & 1,9584 & 1,3616 & 2,2565 & 2,0332 & 2,8402 \\
\hline, 9 & $-0,3673$ & 1,1129 & 0,8775 & 2,0090 & 1,2301 & 2,2976 & 1,8913 & 2,8633 \\
\hline ,8 & $-0,5186$ & 1,2096 & 0,6875 & 2,0446 & 1,0286 & 2,3142 & 1,6677 & 2,8437 \\
\hline, 7 & $-0,6340$ & 1,2684 & 0,5314 & 2,0402 & 0,8603 & 2,2906 & 1,4757 & 2,7832 \\
\hline ,6 & $-0,7307$ & 1,3055 & 0,3919 & 2,0118 & 0,7078 & 2,2422 & 1,2980 & 2,6971 \\
\hline, 5 & $-0,8150$ & 1,3273 & 0,2622 & 1,9648 & 0,5642 & 2,1744 & 1,1274 & 2,5898 \\
\hline, 4 & $-0,8904$ & 1,3363 & 0,1387 & 1,9009 & 0,4258 & 2,0885 & 0,9597 & 2,4625 \\
\hline ,3 & $-0,9587$ & 1,3337 & 0,0190 & 1,8200 & 0,2899 & 1,9841 & 0,7920 & 2,3138 \\
\hline, 2 & $-1,0210$ & 1,3196 & $-0,0987$ & 1,7201 & 0,1544 & 1,8586 & 0,6215 & 2,1403 \\
\hline ,1 & $-1,0777$ & 1,2932 & $-0,2160$ & 1,5972 & 0,0173 & 1,7070 & 0,4450 & 1,9353 \\
\hline 0 & $-1,1290$ & 1,2527 & $-0,3344$ & 1,4435 & $-0,1238$ & 1,5198 & 0,2582 & 1,6862 \\
\hline
\end{tabular}

Algorithme 2 ø Algorithme cumulatif pour l'estimation de $m_{r, \rho}$

INITIALISATION : $\Sigma \leftarrow 0$. Pour $i$ variant de 1 à $N$

CYCLE :

Étape 1 : Produire $z_{1}$ dans l'intervalle $[c, \infty]$, et faire $x_{1} \rightarrow z_{1}$.

Étape 2 : Produire $z_{2}$ et calculer $x_{2}$ selon la transformation (30); si $x_{2}<c$, reprendre à l'étape 1.

Étape 3 : Produire $z_{3}$ et calculer $x_{3}$ selon la transformation (30); si $x_{3}<c$, reprendre à l'étape 1.

Étape $r$ : Produire $z_{r}$ et calculer $x_{r}$ selon la transformation (30); si $x_{r}<c$, reprendre à l'étape 1 .

Étape $r+1$ : Trouver $y=\max \left(x_{1}, x_{2}, \ldots, x_{r}\right)$ et faire $\Sigma \rightarrow \Sigma+y$.

CONCLUSION : Obtenir l'estimé $\hat{m}_{r \rho}(c)=\Sigma / N$, pour une précision d'ordre $1 / \sqrt{N}$.

plan $\left\{\mathrm{X}_{1}>c, \mathrm{X}_{2}>c, \ldots, \mathrm{X}_{k}>c\right\}$, soit :

$\Delta_{k, \rho}^{c}(P)=\int_{c}^{\infty} \int_{c}^{\infty} \cdots \int_{c}^{\infty} x_{1} \cdot f\left(x_{1}, x_{2}, \cdots, x_{k}\right) d x_{k} d x_{k-1} \cdots d x_{1} / h_{k, \rho}(c)$

PATEL et READ (1996, p. 322) rapportent, pour le cas de $(k$ =) 2 variables, l'espérance :

$$
\Delta_{k, \rho}^{c}(P)=\frac{(1+\rho) \phi(c)\left[1-\Phi\left(c \sqrt{\frac{1-\rho}{1+\rho}}\right)\right]}{h_{2, \rho}(c)} .
$$

Notons en outre les relations utiles :

$$
\begin{aligned}
& \Delta_{k, 1}^{c}(P)=\Delta(T)=m_{1}(c) \\
& \Delta_{k, 0}^{c}(P)=\Delta(T)=m_{1}(c),
\end{aligned}
$$

la quantité $m_{1}$ étant définie par (20a) et la constante $c$, selon (8), devant être déterminée d'après la valeur appropriée de $\mathrm{T}$, le taux de qualification par test ou en utilisant (32). Nous n'avons pas élaboré davantage sur ce mode assez particulier de sélection.

\section{Un exemple}

Trois emplois sont ouverts. Supposons que nous disposions aussi de $k=3$ tests, à corrélations mutuelles nulles $(\rho=0)$. Pour faire changement, nous optons de sélectionner le ou les candidats obtenant un score standardisé $\mathrm{X}_{j}>1$, c'est-à-dire $c=1$. Quel bénéfice peut-on escompter d'une telle règle et, accessoirement, quel est le taux de sélection réel?

Le problème d'évaluation du différentiel de sélection est énoncé ici dans une perspective différente de celle adoptée dans l'exposé plus haut. Le lecteur, pour exercer sa compréhension des différents modes du différentiel, est invité à structurer la question et à lui trouver réponse.

Différentiel multiple (de mode A). Nous retenons (dans ce mode) un individu s'il se qualifie au moins dans l'un des 3 tests, donc si l'un de ses 3 scores (en valeurs standardisées) atteint le seuil $c=1$. La probabilité qu'un individu (dans la population de référence) atteigne le seuil $c=1$ pour un test est $\mathrm{T}=1-\Phi(c) \approx 0,15866$. La probabilité qu'il atteigne ce seuil au moins pour 1 test sur 3 s'évalue aisément puisque, avec $\rho=0$, les performances aux trois tests constituent des événements indépendants. Cette pro- 
babilité est un élément du développement binomial : elle correspond au complément de la probabilité que l'individu n'atteigne jamais ce seuil, soit $1-(1-T)^{3} \approx 0,40445$, le taux de sélection global, c.-à-d. la fraction de population dans laquelle les individus sont susceptibles d'être recrutés. Il y aura (en espérance) $\mathrm{T}_{3}=\mathrm{T}^{3} \approx 0,00399$ individus se qualifiant dans les 3 tests, parmi lesquels $\mathrm{T}^{3} / 3 \approx 0,00133$ seraient retenus pour l'emploi $1 ; 2\left(\mathrm{~T}^{2}-\mathrm{T}^{3}\right)=0,04236$ qualifiés seulement dans 2 tests, dont la moitié $(0,02118)$ retenus pour l'emploi 1; enfin, $\mathrm{T}-2\left(\mathrm{~T}^{2}-\mathrm{T}^{3}\right)-\mathrm{T}^{3}=\mathrm{T}-2 \mathrm{~T}^{2}+$ $\mathrm{T}^{3} \approx 0,11231$ qualifiés et retenus seulement pour l'emploi 1. Le score moyen espéré pour ces derniers est $\Delta(\mathrm{T})$ ou $m_{1}(1)$, soit $\phi(c) / T \approx 1,52514$. Pour les individus 2-qualifiés, chacun sera attribué à l'emploi correspondant à son score maximum, d'où le gain escompté est $m_{2,0}(1) \approx 1,776284$ (en appliquant les équations $25-27$ ); de même $m_{3,0}(1) \approx$ 1,91200 pour les individus 3-qualifiés. Le différentiel multiple pour ce cas sera donc

$$
\begin{aligned}
\Delta_{3,0}(c=1) & =[3(0,15866-2 \times 0,02517+0,00399) \times 1,52514 \\
& +3(0,02517-0,00399) \times 1,76284 \\
& +0,00399 \times 1,91200] / 0,40445 \\
& \approx 1,56635
\end{aligned}
$$

Différentiel polyvalent (de mode B). Un individu polyvalent est sélectionné s'il se qualifie dans les trois tests, donc si ses 3 scores atteignent ou débordent le seuil $c=1$. La proportion d'individus qui devraient y parvenir est simplement (pour $\rho=0$ ) $\mathrm{T}_{3}=\mathrm{T}^{3}=0,15866^{3} \approx 0,00399$; il s'agit en effet d'individus rares! Pour autant que chacun des individus 3-qualifiés est attribué à l'emploi pour lequel il a obtenu son score maximum, l'avantage de sélection pour ce mode est (encore) simplement $m_{3,0}(1) \approx 1,91200$, d'où

$$
\Delta_{3,0}^{p}(c=1) \approx 1,9120 .
$$

Différentiel conjoint (de mode C). Retenant encore les individus 3-qualifiés, une infime proportion $\left(\mathrm{T}^{3} \approx 0,00399\right)$ de la population, nous les attribuons maintenant au hasard dans un emploi ou l'autre. Les emplois étant indépendants, le gain espéré dans chaque emploi est simplement (en vertu de $(36 \mathrm{~b})) m_{1}(1) \approx 1,52514$, d'où

$$
\Delta_{3,0}^{c}(c=1) \approx 1,52514 \text {. }
$$

\section{Conclusion}

L'application pratique des différentiels de sélection, qu'ils soient d'un mode ou d'un autre, entraine nécessairement des degrés d'approximation et d'imprécision plus ou moins élevés. Modèle de distribution statistique de la population pour le score à chaque test, ou modèle pour la mesure de performance à l'emploi; valeurs disparates, voire inconnues, des coefficients de validité prédictive $\rho\left(\mathrm{X}_{j}, \mathrm{Y}_{j}\right)$ entre test et mesure de performance; corrélations $\rho\left(\mathrm{X}_{j}, \mathrm{X}_{j}^{\prime}\right)$ inégales entre les tests; taille très petite de l'échantillon de candidats en lice, voilà autant d'éléments qui rendent la précision de nos calculs illusoire.

Un facteur supplémentaire, qui peut sérieusement compromettre la portée des méthodes et calculs exposés ici, est justement l'application réelle, et suivie, des procédures que nous exposons. Dans une grande entreprise qui aurait mis en oeuvre une procédure semblable à une époque antérieure ou pour laquelle les candidats se seraient auto-sélectionnés, les employés présents pourraient présenter pour chaque emploi des caractéristiques différentes, à performance $\mu_{\mathrm{Y}}^{\prime}$ probablement plus élevée que celle $\left(\mu_{\mathrm{Y}}\right)$ de la population virtuelle de candidats. Dans ce contexte, le différentiel de sélection brut, à l'équation (1), ne s'applique plus, et nous aurions en fait affaire à une distribution tronquée de concomitantes (DAVID, 1981), pour le traitement de laquelle les méthodes revues ici ne suffisent pas. Le problème, bien entendu, est pire encore si, au lieu de sélectionner les $100 P \%$ meilleurs candidats de la population dans chaque emploi, on retient par exemple les 5 meilleurs candidats qui se présentent au concours, quels que soient leurs scores : LAURENCELLE (2014) aborde un aspect de ce problème pour le cas de $k=2$ mesures. À défaut de stipuler de sévères hypothèses gouvernant l'échantillonnage de candidats en concours, nous imaginons difficilement une mesure d'efficacité pour ce type de sélection, vraisemblablement le plus répandu dans les entreprises.

Le modèle du différentiel de sélection multiple, avec ses variantes, se heurte donc à des obstacles d'importance qui rendent son implantation ou son application problématiques. Il reste que, croyons-nous, le modèle est intéressant et mérite d'être étudié. De plus, les idées et les méthodes qui l'accompagnent trouveront peut-être chez quelques lecteurs un domaine d'application inopiné, un écho fructueux.

\section{Note de l'auteur}

L'auteur tient à remercier Réal Gélinas, Ph.D., collègue professeur retraité du Département de mathématiques et informatique de l'U.Q.T.R., pour l'aide apportée sur l'équation (16). Une version antérieure de cet article a déjà paru, sous le même titre, dans Lettres Statistiques, 1998, vol. 10, p. 1 à 26, des tableaux révisés ayant paru dans LAURENCELLE (1998), section E : voir Références.

\section{Références}

Anastasi, A. (1988). Psychological testing (6e édition). New York: Macmillan.

BROGDEN, H. E. (1946). On the interpretation of the correlation coefficient as a measure of predictive efficiency. Journal of educational psychology, 37, 65-76. 
BROGDEN, H. E. (1951). Increased efficiency of selection resulting from replacement of a single predictor with several differential predictors. Educational and psychological measurement, 11, 173-195.

Brown, C. W. \& Ghiselli, E. E. (1953). Per cent increase in proficiency resulting from the use of selective devices. Journal of applied psychology, 37, 341-344.

Burrows, P. M. (1972). Expected selection differentials for directional selection. Biometrics, 28(30), 1091-1100.

Burrows, P. M. (1975). Variances of selection differentials in normal samples. Biometrics, 31, 125-133.

Castellan, N. J., Jr. (1966). On the estimation of the tetrachoric correlation coefficient. Psychometrika, 31, 6773.

DAVID, H. A. (1981). Order statistics (2e édition). New York : Wiley.

DEVROYE, L. (1986). Non-uniform random variate generation. New York : Springer-Verlag.

DunNeTt, C. W. (1978). Screening and selection. In W. H. K. et J. M. TANUR (Éd.), International encyclopedia of statistics, vol. 2 (p. 932-936). New York : Free Press.

FINNEY, D. J. (1962). The statistical evaluation of educational allocation and selection. Journal of the Royal Statistical Society B, 24, 525-549.

JARRET, R. F. (1948). Per cent increase in output of selected personnel as an index of test efficiency. Journal of applied psychology, 32, 135-145.

Johnson, N. L., KoTZ, S. \& BALAKRISHnAN, N. (1994). Distributions in statistics : Continuous univariate distributions (2e édition). New York : Wiley.
Johnson, N. L., Kotz, S. \& BALAKRISHnAN, N. (2000). Distributions in statistics : Continuous multivariate distributions (2e édition). New York: Wiley.

Kendall, M. G. \& STUART, A. (1979). The advanced theory of statistics (3 volumes) (4e édition). Macmillan : New York.

LAURENCELLE, L. (1994). Corrélation et décalage de rangs. In L. Laurencelle, R. Bertrand, D. Allaire \& G. CHÂtillon (Éds.), Trois essais de méthodologie quantitative (p. 1-34). Québec (Qc) : Presses de l'Université du Québec.

LAURENCELle, L. (1998). Théorie et techniques de la mesure instrumentale. Québec : Presses de l'Université du Québec.

LAURENCELLE, L. (2001). Hasard, nombres aléatoires et méthode monte carlo. Québec : Presses de l'Université du Québec.

LAURENCELLE, L. (2008). L'étalonnage et la décision psychométrique. Québec: Presses de l'Université du Québec.

LAURENCELLE, L. (2014). Relative selection as a function of two pre-weighted criteria : a study of two procedures. The Quantitative Methods for Psychology, 10, 167-178.

NAGARAJA, H. N. (1982). Some nondegenerate limit laws for the selection differential. Annals of statistics, 10, 13061310 .

OWEN, D. B. \& LI, L. (1980). The use of cutting scores in selection procedures. Journal of educational statistics, 5 , $157-168$.

PATEL, J. K. \& READ, C. B. (1996). Handbook of the normal distribution (2e édition). New York : Marcel Dekker.

\section{Appendice A : Preuve heuristique du théorème (13)}

Pour $k=1$ test, le gain $\Delta_{1}$ touche la fraction de population $\mathrm{T}(=P)$ se qualifiant à ce test, les individus concernés obtenant un score situé dans l'intervalle $(c, \infty)$ et d'espérance $m_{1}$ Le différentiel de sélection est simplement

$$
\Delta_{1}=\left[\mathrm{T} \times m_{1}\right] / P,
$$

en supposant $P=\mathrm{T}$.

Pour $k=2$ tests, nous retrouvons (en espérance) $\mathrm{T}_{2}$ individus se qualifiant aux deux tests à la fois, chacun étant alors assigné selon son score le plus élevé, d'espérance $m_{2}$; par symétrie et en espérance, la demie de $\mathrm{T}_{2}$ sera affectée à chaque test. Pour le test 1 , il reste alors $\left(\mathrm{T}-\mathrm{T}_{2}\right)$ individus assignés à ce test, d'où le gain escompté est

$$
\Delta_{2}=\left[2\left(\mathrm{~T}-\mathrm{T}_{2}\right) m_{1}+\mathrm{T}_{2} m_{2}\right] / P
$$

en supposant $P=2 \mathrm{~T}-\mathrm{T}_{2}$.

Pour $k=3$ tests, le tiers des $\mathrm{T}_{3}$ individus se qualifiant à la fois dans les trois tests est réparti dans le test 1 , avec le score maximum attendu $m_{3}$. Restent $\mathrm{T}-\mathrm{T}_{3}$ individus, dont $\mathrm{T}_{2}-\mathrm{T}_{3}$ sont co-qualifiés dans les tests 1 et 2 , plus $\mathrm{T}_{2}-\mathrm{T}_{3}$ co-qualifiés dans les tests 1 et 3 . La demie de chaque groupe réalisant son score maximum, d'espérance $m_{2}$, dans le test 1 , nous conservons la fraction $T_{2}-T_{3}$ dans ce test. Le contingent résiduel, soit $T-T_{3}-2\left(T_{2}-T_{3}\right)=T-2 T_{2}+T_{3}$, qualifié seulement dans le test 1 , a pour gain spécifique $m_{1}$. Nous enregistrons, au total

$$
\Delta_{3}=\left[3\left(\mathrm{~T}-2 \mathrm{~T}_{2}+\mathrm{T}_{3}\right) m_{1}+3\left(\mathrm{~T}_{2}-\mathrm{T}_{3}\right) m_{2}+\mathrm{T}_{3} m_{3}\right] / P,
$$

en supposant $P=3 \mathrm{~T}-3 \mathrm{~T}_{2}+\mathrm{T}_{3}$.

Par induction, la formule (13) donne la forme générale de ces expressions. 


\section{Citation}

Laurencelle, L. (2015) Le différentiel de sélection multiple. The Quantitative Methods for Psychology, 11(3), $174-188$.

Copyright (C) 2015 Laurencelle. This is an open-access article distributed under the terms of the Creative Commons Attribution License (CC BY). The use, distribution or reproduction in other forums is permitted, provided the original author(s) or licensor are credited and that the original publication in this journal is cited, in accordance with accepted academic practice. No use, distribution or reproduction is permitted which does not comply with these terms.

Received: 18/08/2015 Accepted: 26/08/2015 